\title{
Genetic variation in natural stocks of Sardina pilchardus (sardines) from the western Mediterranean Sea
}

\author{
M. M. RAMON* \& J. A. CASTRO \\ Laboratori de Genètica, Departament de Biologia Fonamental i Ciències de la Salut, Facultat de Ciències, Universitat \\ de les Illes Balears, Palma de Mallorca (Balears), Spain
}

\begin{abstract}
Eleven enzymes coded by 15 loci $\left(A D H^{*}, S O D^{*}, S D H^{*}, L D H-1^{*}, L D H-2^{*}, M E-1^{*}, M E-2^{*}\right.$, $G P I-1^{*}, G P I-2^{*}, I D H^{*}, M D H-1^{*}, M D H-2^{*}, P G M^{*}, A A T^{*}$ and $C K^{*}$ ) were studied using electrophoretic methods in order to characterize stocks of sardines from the western Mediterranean Sea. The results showed differentiated groups distributed throughout the area studied. They did not form a panmictic population, but they existed as semi-independent, although not completely isolated, breeding units with an estimation of migration of $N_{\mathrm{e}} m=3.1$ (number of migrants per generation). In the LDH-1 system a north-south cline was detected. We have also detected a discontinuity between the Alboran and the rest of the Mediterranean populations sampled at the enzymatic level. This result confirms the action of the AlmeriaOran front as a barrier that produces discontinuities as detected in other marine species.
\end{abstract}

Keywords: genetic variation, isozyme polymorphism, sardine, fish stocks.

\section{Introduction}

The use of biological resources requires a knowledge of genetic variability within species. Few species form single homogeneous populations, and fish species are often composed of discrete stocks so that the relationship between them may be independent to a certain degree. The knowledge of the structure of each stock is important for a better commercial exploitation (Carvalho \& Hauser, 1994).

A central problem in studies of fish populations is the definition of the concept of stock, which can be defined in different ways. From a genetic point of view, a stock is a reproductively isolated unit, which is genetically different from other stocks (Jamieson, 1973; Gyllensten, 1985; Ward et al., 1994). The stock unit actually applied in management decisions depends in part on the amount of population data available to assess each stock separately.

Diverse characteristics and methods have been used to analyse stock structure. Allozyme polymorphisms have proven valuable for estimating population divergence and identifying discrete fish stocks. Their utility depends very much on the level of detectable genetic variation (Shaklee, 1983). In

${ }^{*}$ Correspondence: E-mail: dbsmrj0@ps.uib.es several species with little genetic variability (for example squid, Atlantic salmon, sockeye salmon and many lobsters and prawns) biochemical studies need extremely large sample sizes and the dependence of the analysis on a few polymorphic gene loci has been severely criticized by some authors (Shaklee, 1983; Smith et al., 1990). Nevertheless, some species show high genetic variation and in this case, the allozyme polymorphisms have been widely applied (Altukhov \& Salmenkova, 1987; Shaklee et al., 1990; Skaala et al., 1990; Utter, 1991).

Marine fish species show lower levels of genetic differentiation than freshwater or anadromous species, probably owing to less pronounced barriers to migration and gene flow (Gyllensten, 1985; Ward et al., 1994). However, in some cases we can find examples of barriers that restrict migration among stocks to a certain degree. One of these examples is in the Mediterranean Sea. South-eastern Spain is an area widely recognized as a biogeographical border between Mediterranean and Atlantic biotic communities (Rodriguez et al., 1979). The existence of an Almeria-Oran oceanographic front, a well-defined hydrographic boundary between Atlantic and Mediterranean surface waters (Tintoré et al., 1988), is the factor responsible for the current position and maintenance of discontinuities at the allozyme level in 
mussels (Quesada et al., 1995a), oysters (Saavedra et al., 1993) and at the mtDNA level in mussels (Quesada et al., 1995b).

The purpose of this study was the characterization of genetic variation, at the enzymatic level, of the populations of sardines from the western Mediterranean Sea and to reveal the existence of a genetic discontinuity at the allozymic level in the AlmeriaOran front as found in other marine species.

\section{Materials and methods}

\section{Collection of samples}

Samples of Sardina pilchardus were collected by the Instituto Español de Oceanografía (ECOMED90 project, summer of 1990) on the continental platform along the Spanish Mediterranean coast as depicted in Fig. 1. The geographical location and the mean number of fishes (sample size) per locus for each population is indicated in Table 1.

Fish were collected and their heart, liver and ovaries or testes were extracted and immediately frozen on board the cruise ship. Upon arrival at the laboratory they were frozen to $-80^{\circ} \mathrm{C}$ at which they remained until processed by electrophoresis.

\section{Assays}

Samples were analysed by horizontal starch gel electrophoresis. Information about the loci was extracted from the specific literature for fish, and the loci were scored using the same tissue source

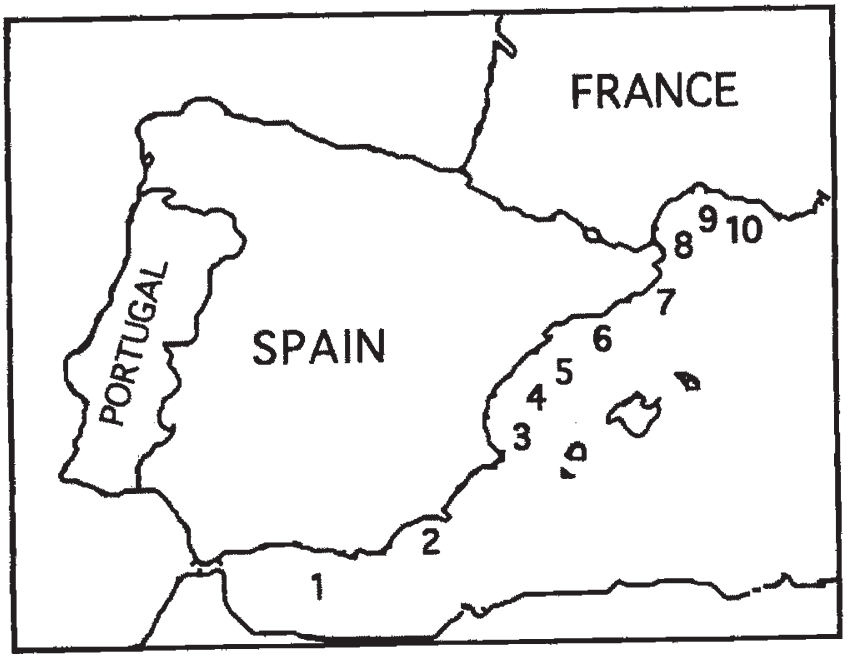

Fig. 1 Samples of Sardina pilchardus collected along the Spanish Mediterranean coast. See Table 1 for an exact geographical location of the captures. (mixture of liver, heart and ovaries and/or testes) and buffer systems. For the study of ADH (alcohol dehydrogenase, EC 1.1.1.1), SOD (superoxide dismutase, EC 1.15.1.1), SDH (sorbitol dehydrogenase, EC 1.1.1.14), LDH (lactate dehydrogenase, EC 1.1.1.27), PGI (glucose-6-phosphate isomerase, EC 5.3.1.9), PGM (phosphoglucomutase, EC 5.4.2.2), and CK (creatine kinase, EC 2.7.3.2) buffers were prepared and samples electrophoresed following the methods described by Ridgway et al. (1970). AAT (aspartate transaminase, EC 2.6.1.1) and $\mathrm{MDH}$ (malate dehydrogenase, EC 1.1.1.37) buffers were prepared following the methods of Clayton \& Tetriak (1972), and those for IDH (isocitrate dehydrogenase, EC 1.1.1.42) and ME (malic enzyme, EC 1.1.1.40) using the methods of Wolf $e t$ al. (1970). Specific staining for each enzyme was performed following methods outlined in Harris \& Hopkinson (1976) and Allendorf et al. (1977).

From the electrophoretic data, allele and genotype frequencies were estimated, and the HardyWeinberg equilibria were tested by the BIOSYS-1 program (Swofford \& Selander, 1989). The Bonferroni procedure was used to correct the deviations in the $\chi^{2}$-test which originated from the number of tests performed (Weir, 1990). The number of alleles per locus, the observed heterozygosity and the proportion of polymorphic loci were calculated for each system and sample.

Patterns of geographical variation were examined in order to detect the existence of clinal variation by means of the Pearson correlation coefficient for arcsine-transformed allele frequencies with geographical distances. To facilitate the comparison between alleles at different frequencies, these were transformed to standardized deviates and expressed in standard deviation units (Quesada et al., 1995a).

The average heterozygosity was evaluated and the data were analysed for heterogeneity by means of $F$-statistics $\left(F_{\text {IS }}, F_{\text {IT }}, F_{\text {ST }}\right)$ (Wright, 1951). Nei's genetic distance (Nei, 1972) was employed for the construction of dendrograms. All these data were calculated with the BIOSYS-1 program (Swofford \& Selander, 1989). The significance of the cluster was tested by means of the bootstrap method, using the programs SEQBOOT, GENDIST, NEIGHBOR and CONSENSE, of the PHYLIP package (Felsenstein, 1993).

\section{Results}

Table 1 shows the parameters of genetic variability in the samples of Sardina. As can be seen, for the proportion of polymorphic loci the range was from 
0.467 (Alicante) to 0.733 (Valencia-1 and Gulf of Lyon-3). With respect to heterozygosity, the range was from 0.110 (Valencia-1) to 0.160 (Gulf of Lyon1 ); the number of effective alleles per locus was around 2 in all samples. The allele frequencies observed in the 10 samples for the 15 enzymatic loci are given in Table 2; the loci, except $L D H-2^{*}$, were polymorphic in some of the samples.

The structure of genetic variation in the sardine samples was evaluated by means of $F$-statistics. Table 3 presents the results of the analysis; the $F_{\text {Is }}$ for the loci show negative values for some of them but they are not statistically significant. The significant values were always positive indicating a lack of equilibrium caused by a deficiency of heterozygotes. The $F_{\text {IS }}$ and $F_{\text {IT }}$ globals (for the loci and the average) displayed positive values and were statistically significant in all systems, with the exception of $\mathrm{LDH}-2^{*}$, thus indicating a deficiency in the number of observed heterozygotes in the samples with respect to the expected number. $F_{\mathrm{ST}}$ was significant for every locus, except $L D H-2^{*}$, and on average. These values indicate that the sardine population in the western Mediterranean is globally structured into different subpopulations, which are genetically differentiated. However, we have to ask if the genetical differentiation was the same for all the populations. To answer this, the $F_{\mathrm{ST}}$ between paired populations was estimated, and the results are in Table 4 . These $F_{\text {ST }}$ values were significant, with some exceptions; but the main thing is that Alboran showed, in general, the highest $F_{\mathrm{ST}}$ compared to the rest of the populations, which clearly indicates the genetical differentiation of this population in relation to the others. To confirm this, a Student's $t$-test between the mean of the $F_{\mathrm{ST}}$ values of Alboran with the other nine populations (column 1 of Table 4) and the mean of the remaining $F_{\mathrm{ST}}$ values was made, and the analysis gave a $t_{8}=4.1 P<0.005$ ), clearly significant. It is important to note that the three samples from the Gulf of Lyon are homogeneous.

The genetic differentiation between the subpopulations was also estimated by means of Nei's genetic distance. The UPGMA dendrogram (Fig. 2a) with all loci and populations sampled, showed one cluster containing the populations of Catalonia and Valencia with the exception of Valencia-3, another cluster with the three Gulf of Lyon populations, and the Alicante and Valencia-3 populations joined to them. The Alboran population with 0.027 units of distance made up the third cluster. The circled numbers are bootstrap per cent values based on 1000 pseudorepetitions. All the bootstrap values were very low, with the exception of Alboran, with 51 per cent. This value, although low, indicated the genetic separation of this population with respect to the others, which together form a single big cluster.

Based on the pattern and magnitude of the allelic frequency variation among the populations, the loci analysed could be classified into two groups. One of them included five loci $\left(S O D^{*}, M D H-2^{*}, P G M^{*}\right.$, $A A T^{*}$ and $C K^{*}$ ) which exhibited the highest variation of frequency based on standard deviation units (mean SDU $=0.115$ ), and the second group was

Table 1 Collection localities and estimates of genetic variability (with standard errors) in samples of Sardina pilchardus from the western Mediterranean Sea. The number of loci scored in all samples was 15 from 11 enzyme systems (see text
for details)

\begin{tabular}{|c|c|c|c|c|c|}
\hline \multirow[b]{2}{*}{ Sample } & \multirow[b]{2}{*}{$\begin{array}{l}\text { Geographical } \\
\text { location }\end{array}$} & \multirow[b]{2}{*}{$\begin{array}{l}\text { Mean } \\
\text { sample size } \\
\text { per locus }\end{array}$} & \multirow[b]{2}{*}{$\begin{array}{l}\text { Mean no. of } \\
\text { alleles per } \\
\text { locus }\end{array}$} & \multicolumn{2}{|c|}{ Proportion of loci } \\
\hline & & & & $\begin{array}{c}\text { Polymorphic* } \\
\text { per } \\
\text { sample }\end{array}$ & $\begin{array}{c}\text { Heterozygous } \\
\text { per } \\
\text { sample }\end{array}$ \\
\hline 1 Alboran & $36^{\circ} 33^{\prime} \mathrm{N}, 03^{\circ} 10^{\prime} \mathrm{W}$ & $41.9 \pm 2.0$ & $1.7 \pm 0.2$ & 0.533 & $0.114 \pm 0.031$ \\
\hline 2 Alicante & $37^{\circ} 32^{\prime} \mathrm{N}, 01^{\circ} 17^{\prime} \mathrm{W}$ & $34.9 \pm 2.1$ & $1.7 \pm 0.1$ & 0.467 & $0.124 \pm 0.036$ \\
\hline 3 Valencia-1 & $39^{\circ} 12^{\prime} \mathrm{N}, 00^{\circ} 05^{\prime} \mathrm{E}$ & $41.2 \pm 1.9$ & $2.1 \pm 0.1$ & 0.733 & $0.110 \pm 0.024$ \\
\hline 4 Valencia-2 & $39^{\circ} 57^{\prime} \mathrm{N}, 00^{\circ} 22^{\prime} \mathrm{E}$ & $42.5 \pm 2.6$ & $2.0 \pm 0.2$ & 0.533 & $0.128 \pm 0.032$ \\
\hline 5 Valencia-3 & $40^{\circ} 23^{\prime} \mathrm{N}, 00^{\circ} 55^{\prime} \mathrm{E}$ & $44.9 \pm 1.5$ & $1.8 \pm 0.1$ & 0.667 & $0.133 \pm 0.027$ \\
\hline 6 Catalonia-1 & $40^{\circ} 54^{\prime} \mathrm{N}, 01^{\circ} 00^{\prime} \mathrm{E}$ & $44.7 \pm 2.5$ & $1.9 \pm 0.1$ & 0.600 & $0.112 \pm 0.020$ \\
\hline 7 Catalonia-2 & $41^{\circ} 31^{\prime} \mathrm{N}, 02^{\circ} 37^{\prime} \mathrm{E}$ & $68.4 \pm 3.6$ & $2.1 \pm 0.2$ & 0.533 & $0.140 \pm 0.035$ \\
\hline 8 Gulf of Lyon-1 & $42^{\circ} 59^{\prime} \mathrm{N}, 03^{\circ} 38^{\prime} \mathrm{E}$ & $45.2 \pm 2.1$ & $1.7 \pm 0.1$ & 0.667 & $0.160 \pm 0.034$ \\
\hline 9 Gulf of Lyon-2 & $43^{\circ} 13^{\prime} \mathrm{N}, 04^{\circ} 21^{\prime} \mathrm{E}$ & $45.0 \pm 1.7$ & $2.0 \pm 0.1$ & 0.600 & $0.148 \pm 0.036$ \\
\hline 10 Gulf of Lyon-3 & $43^{\circ} 16^{\prime} \mathrm{N}, 04^{\circ} 37^{\prime} \mathrm{E}$ & $46.7 \pm 1.7$ & $2.1 \pm 0.1$ & 0.733 & $0.133 \pm 0.020$ \\
\hline
\end{tabular}

*A locus is considered polymorphic when the frequency of the most common allele does not exceed 0.95 . 
Table 2 Allele frequencies for 15 isozyme loci in 10 samples of Sardina pilchardus from the western Mediterranean Sea.

Sample locations are described in Table 1

\begin{tabular}{|c|c|c|c|c|c|c|c|c|c|c|c|}
\hline \multirow[b]{2}{*}{ Locus } & \multirow[b]{2}{*}{ Allele } & \multicolumn{10}{|c|}{ Sample } \\
\hline & & 1 & 2 & 3 & 4 & 5 & 6 & 7 & 8 & 9 & 10 \\
\hline$A D H^{*}$ & $\begin{array}{c}N \\
90 \\
100\end{array}$ & $\begin{array}{c}47 \\
0.032 \\
0.968\end{array}$ & $\begin{array}{c}37 \\
0.122 \\
0.878\end{array}$ & $\begin{array}{c}36 \\
0.069 \\
0.931\end{array}$ & $\begin{array}{l}0^{18} \\
1\end{array}$ & $\begin{array}{c}47 \\
0.117 \\
0.883\end{array}$ & $\begin{array}{c}37 \\
0.027 \\
0.973\end{array}$ & $\begin{array}{c}76 \\
0.033 \\
0.967\end{array}$ & $\begin{array}{c}47 \\
0.128 \\
0.872\end{array}$ & $\begin{array}{c}48 \\
0.167 \\
0.833\end{array}$ & $\begin{array}{c}49 \\
0.122 \\
0.878\end{array}$ \\
\hline$S O D^{*}$ & $\begin{array}{c}N \\
100 \\
130\end{array}$ & $\begin{array}{c}38 \\
0.263 \\
0.737\end{array}$ & $\begin{array}{c}34 \\
0.221 \\
0.779\end{array}$ & $\begin{array}{c}50 \\
0.090 \\
0.910\end{array}$ & $\begin{array}{c}49 \\
0.071 \\
0.929\end{array}$ & $\begin{array}{c}48 \\
0.083 \\
0.917\end{array}$ & $\begin{array}{c}48 \\
0.146 \\
0.854\end{array}$ & $\begin{array}{c}75 \\
0.073 \\
0.927\end{array}$ & $\begin{array}{c}49 \\
0.214 \\
0.786\end{array}$ & $\begin{array}{c}48 \\
0.115 \\
0.885\end{array}$ & $\begin{array}{c}50 \\
0.060 \\
0.940\end{array}$ \\
\hline$S D H^{*}$ & $\begin{array}{c}N \\
90 \\
100\end{array}$ & $\begin{array}{c}48 \\
0.010 \\
0.990\end{array}$ & $\begin{array}{c}20 \\
0.050 \\
0.950\end{array}$ & $\begin{array}{c}49 \\
0.051 \\
0.949\end{array}$ & $\begin{array}{c}31 \\
0.226 \\
0.774\end{array}$ & $\begin{array}{c}29 \\
0.328 \\
0.672\end{array}$ & $\begin{array}{c}12 \\
0.042 \\
0.958\end{array}$ & $\begin{array}{l}31 \\
0 \\
1\end{array}$ & $\begin{array}{c}25 \\
0.100 \\
0.900\end{array}$ & $\begin{array}{c}28 \\
0.125 \\
0.875\end{array}$ & $\begin{array}{c}27 \\
0.037 \\
0.963\end{array}$ \\
\hline$L D H-1^{*}$ & $\begin{array}{c}N \\
80 \\
100\end{array}$ & $\begin{array}{l}0^{46} \\
1\end{array}$ & $\begin{array}{l}50 \\
0 \\
1\end{array}$ & $\begin{array}{c}48 \\
0.010 \\
0.990\end{array}$ & $\begin{array}{l}50 \\
0 \\
1\end{array}$ & $\begin{array}{c}48 \\
0.156 \\
0.844\end{array}$ & $\begin{array}{c}48 \\
0.073 \\
0.927\end{array}$ & $\begin{array}{c}76 \\
0.020 \\
0.980\end{array}$ & $\begin{array}{c}50 \\
0.230 \\
0.770\end{array}$ & $\begin{array}{c}50 \\
0.100 \\
0.900\end{array}$ & $\begin{array}{c}50 \\
0.150 \\
0.850\end{array}$ \\
\hline$L D H-2^{*}$ & $\begin{array}{c}N \\
90 \\
100 \\
120\end{array}$ & $\begin{array}{l}0^{46} \\
1 \\
0\end{array}$ & $\begin{array}{l}50 \\
0 \\
1 \\
0\end{array}$ & $\begin{array}{l}0^{48} \\
0.990 \\
0.010\end{array}$ & $\begin{array}{l}50 \\
0 \\
1 \\
0\end{array}$ & $\begin{array}{l}0^{48} \\
1 \\
0\end{array}$ & \begin{tabular}{l}
\multicolumn{1}{c}{48} \\
0.031 \\
0.969 \\
0
\end{tabular} & $\begin{array}{l}\quad 76 \\
0.020 \\
0.980 \\
0\end{array}$ & $\begin{array}{l}50 \\
0 \\
1 \\
0\end{array}$ & $\begin{array}{l}50 \\
0 \\
1 \\
0\end{array}$ & $\begin{array}{c}50 \\
0.020 \\
0.980 \\
0\end{array}$ \\
\hline$M E-1^{*}$ & $\begin{array}{c}N \\
90 \\
100\end{array}$ & $\begin{array}{c}48 \\
0.146 \\
0.854\end{array}$ & $\begin{array}{l}33 \\
0 \\
1\end{array}$ & $\begin{array}{c}35 \\
0.043 \\
0.957\end{array}$ & $\begin{array}{c}50 \\
0.090 \\
0.910\end{array}$ & $\begin{array}{c}48 \\
0.198 \\
0.802\end{array}$ & $\begin{array}{c}49 \\
0.122 \\
0.878\end{array}$ & $\begin{array}{c}74 \\
0.081 \\
0.919\end{array}$ & $\begin{array}{l}50 \\
0 \\
1\end{array}$ & $\begin{array}{c}44 \\
0.045 \\
0.955\end{array}$ & $\begin{array}{c}50 \\
0.120 \\
0.880\end{array}$ \\
\hline$M E-2^{*}$ & $\begin{array}{c}N \\
95 \\
100\end{array}$ & $\begin{array}{c}48 \\
0.094 \\
0.906\end{array}$ & $\begin{array}{c}36 \\
0.097 \\
0.903\end{array}$ & $\begin{array}{c}35 \\
0.071 \\
0.929\end{array}$ & $\begin{array}{c}50 \\
0.040 \\
0.960\end{array}$ & $\begin{array}{l}0^{48} \\
1\end{array}$ & $\begin{array}{l}0^{49} \\
1\end{array}$ & $\begin{array}{c}74 \\
0.007 \\
0.993\end{array}$ & $\begin{array}{c}50 \\
0.190 \\
0.810\end{array}$ & $\begin{array}{c}44 \\
0.102 \\
0.898\end{array}$ & $\begin{array}{c}50 \\
0.070 \\
0.930\end{array}$ \\
\hline PGI-1* & $\begin{array}{c}N \\
100 \\
140\end{array}$ & $\begin{array}{c}42 \\
0.929 \\
0.071\end{array}$ & $\begin{array}{c}37 \\
0.973 \\
0.027\end{array}$ & $\begin{array}{c}46 \\
0.826 \\
0.174\end{array}$ & $\begin{array}{c}50 \\
0.970 \\
0.030\end{array}$ & $\begin{array}{c}40 \\
0.863 \\
0.137\end{array}$ & $\begin{array}{c}47 \\
0.851 \\
0.149\end{array}$ & $\begin{array}{c}73 \\
0.774 \\
0.226\end{array}$ & $\begin{array}{c}50 \\
0.920 \\
0.080\end{array}$ & $\begin{array}{c}48 \\
0.854 \\
0.146\end{array}$ & $\begin{array}{c}50 \\
0.910 \\
0.090\end{array}$ \\
\hline$P G I-2^{*}$ & $\begin{array}{c}N \\
100 \\
130\end{array}$ & $\begin{array}{l}1^{42} \\
0\end{array}$ & $\begin{array}{c}37 \\
0.986 \\
0.014\end{array}$ & $\begin{array}{c}46 \\
0.924 \\
0.076\end{array}$ & $\begin{array}{c}50 \\
0.970 \\
0.030\end{array}$ & $\begin{array}{l}1^{41} \\
0\end{array}$ & $\begin{array}{c}47 \\
0.968 \\
0.032\end{array}$ & $\begin{array}{l}1^{73} \\
0\end{array}$ & $\begin{array}{l}1^{50} \\
0\end{array}$ & $\begin{array}{c}50 \\
0.990 \\
0.010\end{array}$ & $\begin{array}{c}50 \\
0.970 \\
0.030\end{array}$ \\
\hline$I D H^{*}$ & $\begin{array}{c}N \\
80 \\
90 \\
100 \\
120\end{array}$ & $\begin{array}{l}0^{48} \\
0 \\
1 \\
0\end{array}$ & $\begin{array}{l}0^{20} \\
0 \\
0.975 \\
0.025\end{array}$ & \begin{tabular}{l}
\multicolumn{1}{c}{36} \\
0 \\
0.028 \\
0.972 \\
0
\end{tabular} & \begin{tabular}{l}
\multicolumn{1}{c}{50} \\
0 \\
0.110 \\
0.880 \\
0.010
\end{tabular} & $\begin{array}{l}{ }^{50} \\
0 \\
0.010 \\
0.990 \\
0\end{array}$ & \begin{tabular}{l}
\multicolumn{1}{c}{50} \\
0 \\
0.230 \\
0.770 \\
0
\end{tabular} & $\begin{array}{c}74 \\
0.006 \\
0.223 \\
0.757 \\
0.014\end{array}$ & $\begin{array}{l}{ }^{50} \\
0 \\
0.020 \\
0.980 \\
0\end{array}$ & $\begin{array}{l}{ }^{50} \\
0 \\
0.020 \\
0.970 \\
0.010\end{array}$ & $\begin{array}{c}50 \\
0.010 \\
0.010 \\
0.940 \\
0.040\end{array}$ \\
\hline$M D H-1^{*}$ & $\begin{array}{c}N \\
90 \\
100 \\
120\end{array}$ & $\begin{array}{l}{ }^{30} \\
0 \\
0.917 \\
0.083\end{array}$ & \begin{tabular}{l}
\multicolumn{1}{c}{34} \\
0.015 \\
0.985 \\
0
\end{tabular} & \begin{tabular}{l}
\multicolumn{1}{c}{33} \\
0.076 \\
0.924 \\
0
\end{tabular} & \begin{tabular}{l}
\multicolumn{3}{c}{33} \\
0.030 \\
0.970 \\
0
\end{tabular} & $\begin{array}{c}41 \\
0.098 \\
0.793 \\
0.109\end{array}$ & \begin{tabular}{l}
\multicolumn{1}{c}{50} \\
0.150 \\
0.850 \\
0
\end{tabular} & \begin{tabular}{l}
\multicolumn{1}{l}{72} \\
0.125 \\
0.875 \\
0
\end{tabular} & $\begin{array}{l}\quad 46 \\
0.152 \\
0.848 \\
0\end{array}$ & \begin{tabular}{l}
\multicolumn{1}{c}{46} \\
0.033 \\
0.967 \\
0
\end{tabular} & $\begin{array}{c}50 \\
0 \\
0.910 \\
0.090\end{array}$ \\
\hline$M D H-2^{*}$ & $\begin{array}{c}N \\
80 \\
100 \\
110 \\
\text { null }\end{array}$ & \begin{tabular}{l}
\multicolumn{1}{c}{27} \\
0.093 \\
0.722 \\
0 \\
0.185
\end{tabular} & $\begin{array}{l}33 \\
0 \\
1 \\
0 \\
0\end{array}$ & \begin{tabular}{l}
\multicolumn{1}{c}{33} \\
0.091 \\
0.879 \\
0 \\
0.030
\end{tabular} & \begin{tabular}{l}
\multicolumn{1}{c}{33} \\
0.061 \\
0.939 \\
0 \\
0
\end{tabular} & $\begin{array}{l}0^{41} \\
1 \\
0 \\
0\end{array}$ & \begin{tabular}{l}
\multicolumn{1}{c}{45} \\
0.133 \\
0.867 \\
0 \\
0
\end{tabular} & $\begin{array}{l}{ }^{72} \\
0 \\
0.972 \\
0.028 \\
0\end{array}$ & $\begin{array}{l}0^{47} \\
1 \\
0 \\
0\end{array}$ & $\begin{array}{l}46 \\
0 \\
1 \\
0 \\
0\end{array}$ & $\begin{array}{c}47 \\
0 \\
0.968 \\
0.032 \\
0\end{array}$ \\
\hline$P G M^{*}$ & $\begin{array}{c}N \\
100 \\
140\end{array}$ & $\begin{array}{l}1^{46} \\
0\end{array}$ & $\begin{array}{c}29 \\
0.638 \\
0.362\end{array}$ & $\begin{array}{c}30 \\
0.550 \\
0.450\end{array}$ & $\begin{array}{c}47 \\
0.500 \\
0.500\end{array}$ & $\begin{array}{c}45 \\
0.889 \\
0.111\end{array}$ & $\begin{array}{c}43 \\
0.558 \\
0.442\end{array}$ & $\begin{array}{c}66 \\
0.803 \\
0.197\end{array}$ & $\begin{array}{c}46 \\
0.804 \\
0.196\end{array}$ & $\begin{array}{c}45 \\
0.778 \\
0.222\end{array}$ & $\begin{array}{c}50 \\
0.860 \\
0.140\end{array}$ \\
\hline$A A T^{*}$ & $\begin{array}{c}N \\
50 \\
75 \\
100\end{array}$ & $\begin{array}{l}0^{28} \\
0.375 \\
0.625\end{array}$ & \begin{tabular}{l}
\multicolumn{1}{c}{37} \\
0 \\
0.230 \\
0.770
\end{tabular} & $\begin{array}{l}0^{47} \\
0.319 \\
0.681\end{array}$ & $\begin{array}{c}37 \\
0.014 \\
0.108 \\
0.878\end{array}$ & \begin{tabular}{l}
\multicolumn{1}{c}{51} \\
0 \\
0.167 \\
0.833
\end{tabular} & $\begin{array}{l}0^{50} \\
0.040 \\
0.960\end{array}$ & $\begin{array}{c}75 \\
0.007 \\
0.280 \\
0.713\end{array}$ & $\begin{array}{l}{ }^{4} 40 \\
0.200 \\
0.800\end{array}$ & $\begin{array}{c}31 \\
0.016 \\
0.194 \\
0.790\end{array}$ & $\begin{array}{c}38 \\
0 \\
0.224 \\
0.776\end{array}$ \\
\hline$C K^{*}$ & $\begin{array}{c}N \\
85 \\
100 \\
120\end{array}$ & \begin{tabular}{l}
\multicolumn{1}{c}{44} \\
0.614 \\
0.386 \\
0
\end{tabular} & \begin{tabular}{l}
\multicolumn{1}{l}{36} \\
0.528 \\
0.472 \\
0
\end{tabular} & \begin{tabular}{l}
\multicolumn{1}{c}{46} \\
0.196 \\
0.804 \\
0
\end{tabular} & $\begin{array}{c}40 \\
0.500 \\
0.488 \\
0.012\end{array}$ & \begin{tabular}{l}
\multicolumn{1}{c}{49} \\
0.296 \\
0.704 \\
0
\end{tabular} & $\begin{array}{l}\quad 47 \\
0.245 \\
0.755 \\
0\end{array}$ & \begin{tabular}{l}
\multicolumn{1}{c}{39} \\
0.308 \\
0.692 \\
0
\end{tabular} & \begin{tabular}{l}
\multicolumn{1}{c}{28} \\
0.464 \\
0.536 \\
0
\end{tabular} & $\begin{array}{l}\quad 47 \\
0.277 \\
0.723 \\
0\end{array}$ & $\begin{array}{c}39 \\
0.526 \\
0.474 \\
0\end{array}$ \\
\hline
\end{tabular}

$N$, number of individuals.

(C) The Genetical Society of Great Britain, Heredity, 78, 520-528. 


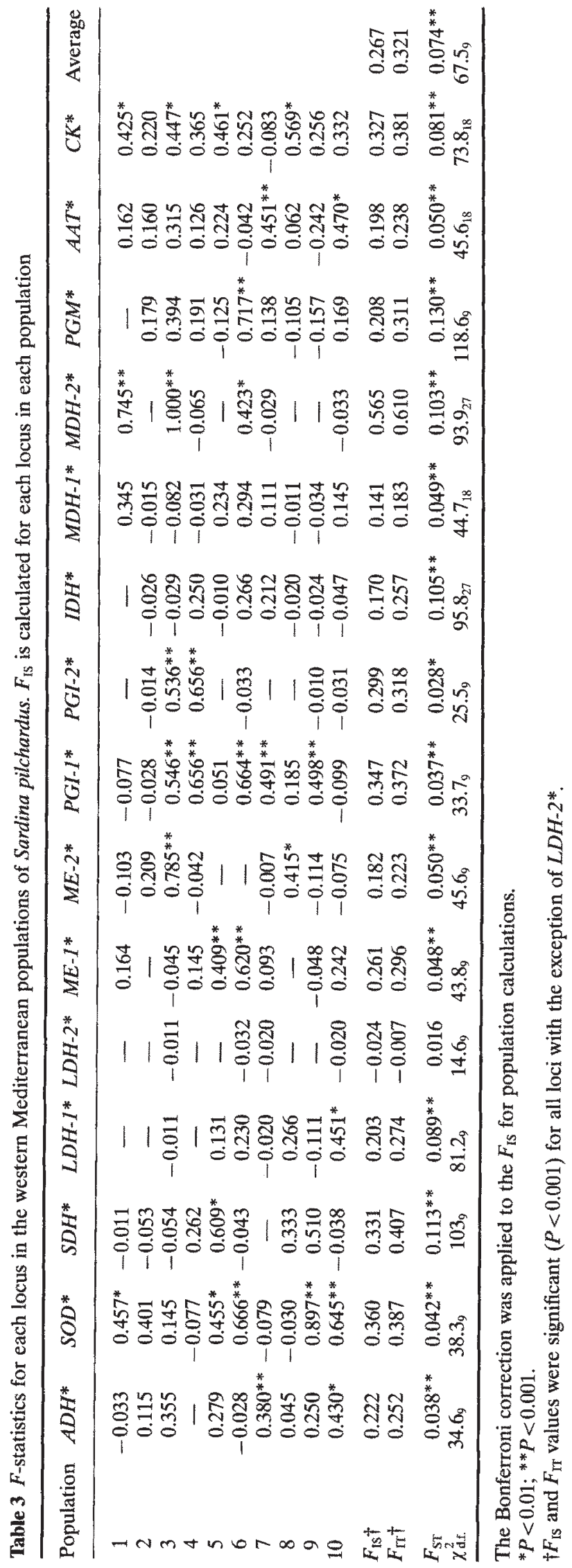


Table $4 F_{\mathrm{ST}}$ betweenpairedpopulationsofSardinapilchardus. ThenumbersindicatethepopulationsasdescribedinTable1

\begin{tabular}{rrrrccccccc}
\hline \multicolumn{10}{c}{ Population } \\
\cline { 3 - 11 } Population & $N$ & 1 & 2 & 3 & 4 & 5 & 6 & 7 & 8 & 9 \\
\hline 1 & 42 & - & - & - & - & - & - & - & - & - \\
2 & 35 & $0.051^{* *}$ & - & - & - & - & - & - & - & - \\
3 & 41 & $0.082^{* * *}$ & $0.036^{*}$ & - & - & - & - & - & - & - \\
4 & 39 & $0.088^{* * *}$ & $0.026 \mathrm{NS}$ & $0.040^{*}$ & - & - & - & - & - & - \\
5 & 45 & $0.070^{* * *}$ & $0.060^{* *}$ & $0.053^{* *}$ & $0.055^{* *}$ & - & - & - & - & - \\
6 & 45 & $0.096^{* * *}$ & $0.049^{* *}$ & $0.036^{*}$ & $0.032^{*}$ & $0.051^{* *}$ & - & - & - & - \\
7 & 69 & $0.061^{* * *}$ & $0.044^{* *}$ & $0.028^{*}$ & $0.052^{* * *}$ & $0.041^{* *}$ & $0.028^{*}$ & - & - & - \\
8 & 42 & $0.047^{* *}$ & $0.022 \mathrm{NS}$ & $0.045^{*}$ & $0.041^{* *}$ & $0.033^{*}$ & $0.048^{* *}$ & $0.039^{* *}$ & - & - \\
9 & 45 & $0.061^{* *}$ & $0.026^{*}$ & $0.021 \mathrm{NS}$ & $0.041^{* *}$ & $0.021 \mathrm{NS}$ & $0.034^{*}$ & $0.022^{*}$ & $0.016 \mathrm{NS}$ & - \\
10 & 47 & $0.033^{*}$ & $0.025^{*}$ & $0.048^{* *}$ & $0.044^{* *}$ & $0.030^{*}$ & $0.052^{* *}$ & $0.029^{* *}$ & $0.016 \mathrm{NS}$ & $0.019 \mathrm{NS}$ \\
\hline
\end{tabular}

${ }^{*} P<0.05,{ }^{*} P<0.005,{ }^{* *} P<0.001 ; \mathrm{NS}$, not significant; $N$, number of individuals.

(a)

$0.05 \quad 0.03 \quad 0,02 \quad 0,00$

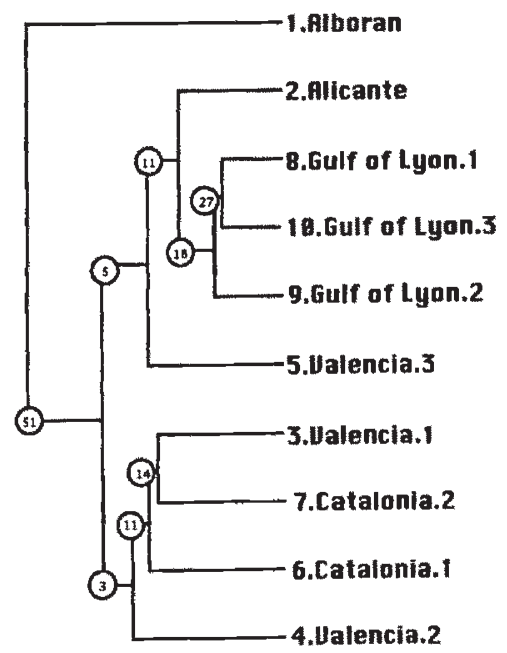

(b)
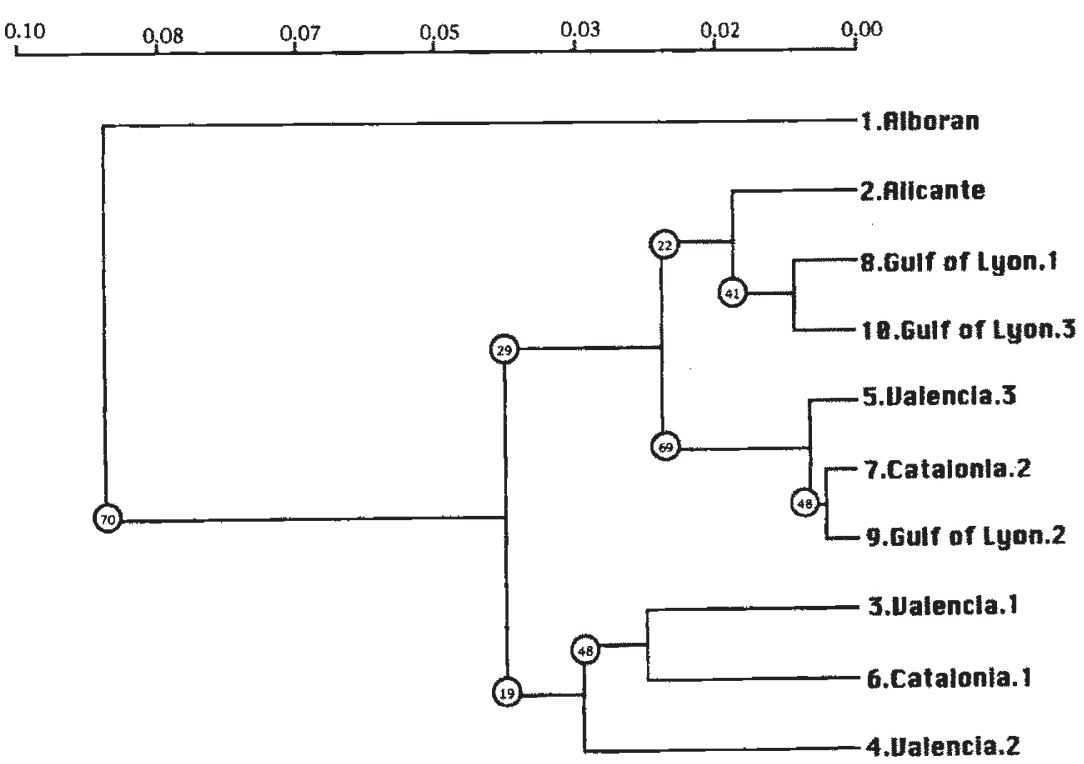

Fig. 2 UPGMA dendrogram for Sardinia pilchardus based on the Nei (1972) genetic distance. (a) With all loci. Cophenetic correlation was 0.732 . (b) With only the five discriminatory enzymes. Cophenetic correlation was 0.797.

formed by the rest of the loci, with minor variation of frequency (mean SDU $=0.065$ ). A MannWhitney U-test between these two was significant (Sokal \& Rohlf, 1981). A new cluster was based on Nei's distance with these five variable loci and a bootstrap analysis of this cluster was undertaken (Fig. 2b). This cluster confirmed the Alboran separation and increased the bootstrap percentage value of this separation with respect to the other populations to 70 per cent, which was a more significant value than that based on all the enzymes.

\section{Discussion}

We have used electrophoretic traits to analyse the genetic differences that may exist among the samples of sardine in the western Mediterranean Sea. Although the results obtained for other fish 
species in the literature are not directly comparable to the populations of sardines, we have detected more variability than described in the other species. Thus, most enzymatic systems studied in sardine populations are polymorphic, in contrast to studies of other fish species (Ryman, 1983; Wilmot \& Burger, 1985; Smith, 1986). This could be a result, in part, of the allozymic loci chosen in this study, but we have found that some loci which are polymorphic in our sardine population study were monomorphic in other species of fishes, for example: $A D H^{*}, I D H^{*}$, $P G I^{*}$ and $C K^{*}$ in the Atlantic Salmon (Stähl, 1987), $C K^{*}, M D H^{*}, P G I^{*}$ and $S O D^{*}$ in sockeye Salmon (Wilmot \& Burger, 1985) and $L D H^{*}$ and $P G I^{*}$ in anchovy and sardine populations (Spanakis et al., 1989).

A high level of heterogeneity in the Mediterranean population of sardines was shown when the genetic variation was measured as observed heterozygosity, which ranged from 0.110 to 0.160 . This range is higher than marine fish data reported by Gyllensten (1985) and Ward et al. (1994) who found similar values of average total heterozygosity (0.063 in six species and 0.064 in 54 species, respectively).

The proportion of genetic diversity attributable to differences among subpopulations can be measured by $F_{\mathrm{ST}}$. More than 60 per cent of marine species show a low population differentiation $\left(G_{\mathrm{ST}}<0.05\right)$ (Ward et al., 1994), although significant population differences in some of these species have been detected (Smith et al., 1990). In the sardine population we have estimated $F_{\mathrm{ST}}=0.074$, which is similar to the $G_{\mathrm{ST}}$ value postulated by Ward et al. (1994) (0.062) and bigger than the 0.042 described by Gyllensten (1985). Following Ward et al. (1994) a $G_{\text {ST }}>0.02$ means that the population is structured. In this way, we can say that the population of the western Mediterranean sardine is structured into genetically differentiated subpopulations.

Alternatively, $F_{\text {ST }}$ can be used to derive an estimation of $N_{\mathrm{e}} m$, a reflection of the number of migrants per generation. Using this approach, $N_{\mathrm{e}} m$ was estimated at 3.1. The $N_{\mathrm{e}} m$ estimated by Ward et al. (1994) for freshwater and marine fish corresponds to 0.88 and 3.78 , respectively, this latter value being very similar to that found by us in the sardine populations, indicating a similar level of migration.

In the present study the mean heterozygosity observed is lower than expected, as indicated by the global $F_{\mathrm{IS}}$, where the positive values indicate a deficiency in the heterozygotes. This could be a result of inbreeding of genetic cohorts in the samples with the existence of sib relatives, or it could also be a result of the inclusion of more than one genetically differentiated cohort in a single sample.

We have posed two main questions: (i) can the population of sardines in the western Mediterranean be considered as a unique population or, on the contrary, are the samples genetically independent of each other? and (ii) has the Almeria-Oran front affected the populations of sardines?

With respect to the first question, fish species tend to be subdivided into genetically different subpopulations or stocks. Chabanaud (1926) and Lee (1962) recognized two stocks of sardines, one in the Atlantic (Sardina pilchardus pilchardus) and another in the Mediterranean (Sardina pilchardus sardina), as different species, but Svetovidov (1964) considered them only as different races, in accordance with the observations of Furnestin (1952), who also claimed the existence of different races within the Atlantic stock. In the Mediterranean, Laskaridis (1948) recognized four groups in the Greek seas, and Skrivanic \& Zavodnik (1973) supported the view that in the Adriatic Sea two major populations of sardines can be found, one in the north and the other in the south, based mainly on hydrographic features of the Adriatic. Spanakis et al. (1989) suggest, in a genetic study concerning Aegean and Ionian populations of sardines, that they do not form an undifferentiated panmictic population, but they exist as semi-independent but not completely isolated breeding units. Our results are in accordance with their hypothesis.

With respect to the second question, the cluster analysis detected a clear displacement of the Alboran population with respect to the rest of the populations; and the $F_{\mathrm{ST}}$ between paired populations (Table 4) showed a clear significant difference of Alboran in respect to the other samples. In this area, the mussel Mytilus galloprovincialis exhibits an abrupt discontinuity in allozyme frequencies (Quesada et al., 1995a) which appears to be associated with the Almeria-Oran oceanographic current front (Tintoré et al., 1988), a zone of turbulence in the Alboran Sea, which also may restrict larval dispersal in the oyster Ostrea edulis (Saavedra et al., 1993). Recently, Alemany \& Alvarez (1993) studied the same samples of sardines from the ECOMED90 programme, although they compared growth parameters. They observed significant growth differences, greater in the Alboran area than in the other populations and suggested that sardine populations of the Alboran area could belong to the Atlantic subspecies. The authors explained these differences by the action of environmental factors. Of these, mainly upwelling produced by the flux of Atlantic 
waters or winds and variation of winter surface temperatures from the Alboran sea to the Gulf of Lyon would be directly related to sardine growth values; although they did not disregard the action of genetic factors, supported by a lack of significant migration. All of these factors are related to the action of Almeria-Oran front.

In order to prove a possible relationship between the genetic variation and geographical distribution we tested the correlation between genetic variation (gene frequencies, heterozygosity) and latitude. Only for the frequency of alleles of $L D H-I^{*}$ did we detect the existence of a cline with an increasing northsouth distribution, as can be seen in Fig. 3. The clinal distribution for $L D H-1^{*}$ agrees with the significance of allelic isozyme variation postulated by Powers et al. (1991). These authors detected two alleles for $L D H-B$ in Fundulus heteroclitus with allele $b$ predominant in the northern (colder) cline and $a$ predominant in the southern (warmer) cline. The two isozymes differ in catalytic efficiency, concentration and stability of enzyme. These differences affected the rate of development during the first days of embryonic growth.

With respect to the forces that have established and maintained the differentiation in the population of sardine, apart from the Almeria-Oran front, we think that an initial founder effect, followed by genetic drift within each stock, the existence of genetic flux but lack of significant migration along the coast and environmental adaptations, could be relevant. Although natural selection could act on the $L D H-1^{*}$ polymorphism, functional nonequivalence of allelic products is demonstrated, and it is not

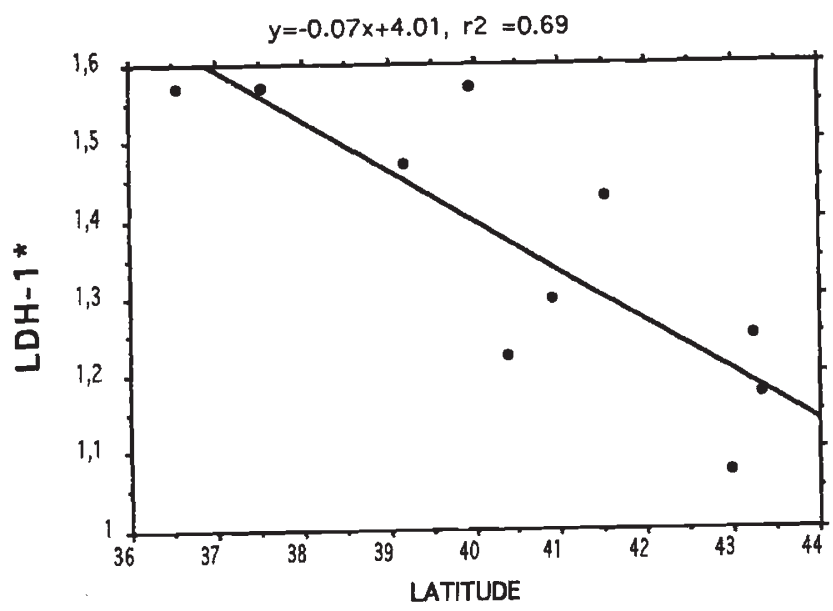

Fig. 3. Regression of the $L D H-]^{*}$ allele frequency and latitude in the different samples of sardines. The allele frequencies have the transformation $\arcsin (\sqrt{ } \mathrm{p})$. The regression is significant, $P=0.003$. known how selection acts at the molecular level. In relation to the five loci which characterize the separation of the Alboran and the other populations, we do not know how these enzymes could act in this discrimination, and an in-depth study with these enzymes in the future would be necessary to solve this issue.

\section{Acknowledgements}

We thank A. Bennasar, O. Reñones, B. Amengual and Dr P. Oliver of the Instituto Español de Oceanografía (Laboratory of Palma de Mallorca) for their collaboration in obtaining and treating the samples and M. J. Arranz for technical assistance. We also thank Dr G. Alvarez for useful comments on the manuscript.

\section{References}

AlEMANy, F. AND AlVAREZ, F. 1993. Growth differences among sardine (Sardina pilchardus Walb.) populations in Western Mediterranean. Sci. Mar., 57, 229-234.

ALLENDORF, F. W., MITCHELL, N., RYMAN, N. AND STÄHL, G. 1977. Isozyme loci in brown trout (Salmo trutta L.): detection and interpretation from population data. Hereditas, 86, 179-190.

Altukhov, Y. P. AND SAlmenkova, E. A. 1987. Stock transfer relative to natural organization, management and conservation of fish populations. In: Ryman, N. and Utter, F. (eds) Population Genetics and Fishery Management, pp. 333-344. University of Washington Press, Seattle.

CARVAlHO, G. R. AND HAUSER, L. 1994. Molecular genetics and the stock concept in fisheries. Rev. Fish Biol. Fish., 4, 326-350.

Chabanaud, P. 1926. Sur les clupeides du genre Sardina et de divers genres voisins. Bull. Soc. Zool. France, 51, $8-16$.

Clayton J. W. AND TETRIAK, D. N. 1972. Amine-citrate buffers for $\mathrm{pH}$ control in starch gel electrophoresis. $J$. Fish. Res. Board Can., 29, 1169-1172.

FELSENSTEIN, J. 1993. PHYLIP (Phylogeny Inference Package). Version 3.5c. Univ. of Washington, Seattle.

FURNestin, J. 1952. Biologie de clupeides mediterranées. Journées d'Etudes du Laboratoire Arago (Suppl. No. 3 a Vie et Milieu), 2, 96-116.

GYLLENSTEN, U. 1985. The genetic structure of fish: differences in the intraspecific distribution of biochemical genetic variation between marine, anadromous, and freshwater species. J. Fish Biol., 26, 691-699.

HARRIS, H. AND HOPKINSON, D. A. 1976. Handbook of Enzyme Electrophoresis in Human Genetics. NorthHolland Publishing Co., Amsterdam, The Netherlands. JAMIESON, A. 1973. Genetic 'tags' for marine fish stocks. In: Hardin, J. F. R. (ed.) Sea Fisheries Research, pp. 91-99. Elek Science, London. 
LASKARIDIS, K. 1948. Study of the biology of sardine (Clupea pilchardus Walb.) in Greek waters. Prakt. Hell. Hydrobiol. Fish. Bull., 85, 561-568.

LEE, J. Y. 1962. Sardines de l'Atlantique et sardines de la Mediterranée. Différences morphologiques, biologiques et sérologiques. Conseil International pour l'Exploration de la Mer. Council Meeting. Comité de la Sardine. No. 14, 1-5.

NEl, M. 1972. Genetic distance between populations. Am. Nat., 106, 283-292.

POWERS, D. A., LAUERMAN, T., CRAWFORD, D., SMITH, M., GONZALEZ-VILLASENOR, 1. AND DIMICHELE, L. 1991. The evolutionary significance of genetic variation at enzyme synthesizing loci in the teleost Fundulus heteroclitus. J. Fish Biol., 39 (Suppl. A), 169-184.

QuesadA, H., ZaPATA, C. AND Alvarez, G. 1995a. A multilocus allozyme discontinuity in the mussel Mytilus galloprovincialis: the interaction of ecological and life-history factors. Mar. Ecol. Progr. Ser, 116, 99-115.

QUESADA, H., BEYNON, C. M. AND SKIBINSKI, D. O. F. 1995 b. A mitochondrial DNA discontinuity in the mussel Mytilus galloprovincialis Lmk: Pleistocene vicariance biogeography and secondary intergradation. Mol. Biol. Evol., 12, 521-524.

RIDGWAY, G. J., SHERburNe, S. W. AND LEWIS, R. D. 1970. Polymorphism in the esterases of Atlantic herring. Trans. Am. Fish. Soc., 99, 147-151.

RODRIGUEZ, J., GARCIA, A. AND RODRIGUEZ, v. 1979. Zooplanktonic communities of the divergence zone in the northwestern Alboran Sea. Mar Ecol., 3, 133-142.

RYMAN, N. 1983. Patterns of distribution of biochemical genetic variation in salmonids: differences between species. Aquaculture, 33, 1-21.

SAAVEDRA, C., ZAPATA, C., GUERRA, A. AND ALVAREZ, G. 1993. Allozyme variation in European populations of the oyster Ostrea edulis. Mar. Biol., 115, 85-95.

SHAKLEE, J. B. 1983. The utilization of isozymes as gene markers in fisheries management and conservation. Isozymes: Curr. Top. Biol. Med. Res., 11, 213-247.

SHAKLEE, J. B., PHELPS, S. R. AND SALinı, J. 1990. Analysis of fish stock structure and mixed-stock fisheries by electrophoretic characterization of allelic isozymes. In: Whitmore, D. H. (ed.) Electrophoretic and Isoelectric Focusing Techniques in Fisheries Management, pp. 173-196. CRC Press, Boca Raton FL.

SKAALA, O., DAHLE, G., JøRSTAD, K. AND NÆVDAL, G. 1990. Interactions between natural and farmed fish populations: information from genetic markers. J. Fish Biol., 36, 449-460.

SKRIVANIC, A. AND ZAVODNIK, D. 1973. Migration of the sardine (Sardina pilchardus) in relation to hydrographical conditions of the Adriatic Sea. Neth. J. Sea Res., 7, 7-18.

SMITH, P. J. 1986. Genetic similarity between samples of the orange roughly Hoplostethus atlanticus from the Tasman Sea, south-west Pacific Ocean and north-east Atlantic Ocean. Mar. Biol., 91, 173-180.

SMITH, P. J., JAMIESON, A. AND BIRLEY, A. J. 1990. Electrophoretic studies and stock concept in marine teleosts. $J$. Cons. int. Explor. Mer, 47, 231-245.

SOKAL, R. R. AND ROHLF, F. J. 1981. Biometry, 2nd edn. W. H. Freeman and Company, New York.

SPANAKIS, E., TSIMENIDES, N. AND ZOUROS, E. 1989. Genetic differences between populations of sardine, Sardina pilchardus, and anchovy, Engraulis encrasicolus, in the Aegean and Ionian Seas. J. Fish Biol., 35, 417-437.

STÄHL, G. 1987. Genetic population structure of Atlantic Salmon. In: Ryman, N. and Utter, F. (eds) Population Genetics and Fishery Management, pp. 121-140. University of Washington Press, Seattle.

SVerovidov, A. N. 1964. Rydy Tchernogo More. Zoologicheskii Institut Akademii Nauk SSSR. MoscowLeningrad.

SWOFFORD, D. L. AND SElANDER, R. B. 1989. BIOSYs-1. $A$ computer program for the analysis of allelic variation in population genetics and biochemical systematics. Release 1.7. University of Illinois, Urbana, IL.

TINTORÉ, J., LA VIOLETTE, P. E., BLADE, 1. AND CRUZADO, G. 1988. A study of an intense density front in the eastern Alboran Sea: the Almería-Oran front. J. Phys. Oceanogr., 18, 1384-1397.

UTTER, F. M. 1991. Biochemical genetics and fishery management: an historical perspective. J. Fish Biol., 39 (Suppl. A), 1-20.

WARD, R. D., WOODWARK, M. AND SKIBINSK1, D. O. F. 1994. A comparison of genetic diversity levels in marine, freshwater, and anadromous fishes. J. Fish Biol., 44, 213-232.

WElR, B. S. 1990. Genetic Data Analysis. Sinauer Associates, Sunderland, MA.

WILMOT, R. L. AND BURGER, C. v. 1985. Genetic differences among populations of Alaskan sockeye salmon. Trans. Am. Fish. Soc., 114, 236-243.

WOLF, V., ENGEL, w. AND FAUST, J. 1970. Zum Mechanismus der Diploidisierung in der Wirbeltierevolution: Koexistenz von tetrasomen und disomen Genloci der Isocitrat-dehydrogenasen bei der Regenbogenforelle (Salmo irideus). Humangenetik, 9, 150-156.

WRIGHT, s. 1951. The genetical structure of populations. Ann. Eugen., 15, 323-354. 